\title{
CORRECTIONS
}

\section{US revamps domestic Ebola response}

In the third paragraph of this Feature (BMJ 2014;349:g6417, doi:10.1136/bmj.g6417), we should have listed Liberia, Guinea, and Sierra Leone (not "Senegal," as was published) as the three

Cite this as: BMJ 2014;349:96530

west African countries affected by Ebola. 